\title{
A Survey of Multi-Agent based Intelligent Decision Support System for Medical Classification Problems
}

\author{
Hanaa Salem \\ Communications \& Computer \\ Dept. \\ Faculty of Engineering \\ Delta University, Egypt
}

\author{
Gamal Attiya \\ Computer Science \& \\ Engineering Dept. \\ Faculty of Electronic \\ Engineering \\ Menoufia University, Egypt
}

\author{
Nawal El-Fishawy \\ Computer Science \& \\ Engineering Dept. \\ Faculty of Electronic \\ Engineering \\ Menoufia University, Egypt
}

\begin{abstract}
There has been growing on big data since last decade for discovering useful trends or patterns that are used in diagnosis and decision making. Intelligent decision support system an automated judgment that supports decision making is composed of human and computer interaction to help in decision making accuracy. Also multi-agent systems (MAS) are collections of independent intelligent entities that collaborate in the joint resolution of a complex problem. Multi-agent intelligent decision support systems can be used to solve large-scale convention problem. This paper is a survey of the recent research in multiagent and intelligent decision support systems to support for classification problems. The purpose of the survey described in this paper is the development of a novel large-scale hybrid medical diagnosis system based on Multi-agent Intelligent Decision Support System (IDSS) for distributed database.
\end{abstract}

\section{Keywords}

Multi-Agent; Intelligent Decision Support Systems; Diagnosis; Feature Selection

\section{INTRODUCTION}

Medical diagnosis is known to be subjective for several reasons: Firstly it relies on the physician making the diagnosis. Secondly, and most essentially, the measure of information that should be analyzed to make a good prediction is usually huge and at times uncontrollable. Machine learning can be utilized to automatically conclude diagnostic rules from descriptions of past, successfully treated patients, and help experts and specialists make the diagnostic process more objective and more reliable. An Intelligent decision support systems are defined as interactive computer systems used to help decision making to use data sets and models in order to find problems, solve problems and take decisions so dataset and models are designed to assist in decision making through a semi-structured and unstructured decision making modules that support for decision making [1]. A definition for the using of the computer technologies in assistant diagnose, which is: 'Medical artificial intelligence is primarily concerned with the construction of AI programs that perform diagnosis and make therapy recommendations. Unlike medical applications built on other programming approaches, such as morally statistical and probabilistic approaches, medical AI programs are based on representative models of disease units and their relationship to patient factors. Recently, many studies focus on intelligent decision support system rather than the Artificial Intelligence in Medicine (AIM) systems [2].

Many studies in the use of the agents in medical classification approaches represent the latest research direction, which intends to disregard disadvantages of earlier developed medical classification problems computational systems (medical expert systems for example), that usually consist in limited autonomy, interaction ability with the environment and intelligence in the problem solving. Important applications of the agents and multiagent systems are represented in diverse problem solving that appear in medical classification problems.

Examples of medical problems that can be resolved by artificial agents, medical diagnostics elaboration, medical data gatherings about patients, medical knowledge search, medical decisions support, pro-active assistance of the physicians [3]. Agent-based intelligent decision support systems (IDSS) to support decision making is significant within the medical classification problems because they let doctors and nurses to rapidly gather information and process it in numerous ways in order to support with making diagnosis and treatment decisions. These frameworks deigned to support mainly in medical areas that is varied from the sorting and recovery of medical records, storing and recovery of key substances in medicines, analysis of real-time information accumulated from screens, analysis of X-Rays, analysis of patient history for the purposes of diagnosis, study of family history (for cardiac conditions for example), and in many other areas [4]. These frameworks condescended to bolster for the most part in therapeutic ranges that is assorted from the putting away and recovery of restorative records, putting away and recovery of key substances in pharmaceuticals, investigation of ongoing information accumulated from screens, examination of X-Rays, investigation of patient history for the reasons of analysis, investigation of family history (for cardiovascular conditions for instance), and in numerous different zones.

This paper is directed to highlight the current research in IDSS and multi-agent. This will involve looking at multi-agents, intelligent decision support systems (IDSS) and current Multiagent both inside and outside the classification medical data. The information gathered in this research will help with identifying potential issues with multi-agent based IDSS particularly with our intended future work on Multi-Agent based IDSS for medical dataset classification.

\section{RELATED WORK}

\subsection{Overview of Intelligent Decision Support Systems}

In medical industry, the use of intelligent decision support systems (IDSS) to support decision making is necessary and important because they allow doctors and nurses to rapidly collect information and process it in numerous ways in order to support with making diagnosis and treatment decisions.

Intelligent decision support system (IDSS) is a result of combining decision support system (DSS) and artificial intelligent (AI). Its basic design thinking is to combine the 
knowledge reasoning techniques of $\mathrm{AI}$ and the basic function models of DSS [5]. The benefits of using intelligent components with DSSs as opposed to plain IDSSs to improve decisions making timeliness, consistency in decisions, explanations and justifications for specific recommendations, enhanced management of uncertainty, and formalization of organizational knowledge. The greatest useful of these advantages is the enhanced explanations and justifications which is very much indeed useful feature particularly in field like medicine, where it helps if the real expert can authenticate the machines reasoning [6]. Artificial Intelligence approaches have been used in intelligent decision support systems to: 1) aid physicians in their diagnosis techniques; 2) make decisions more exact, accurate and effective, 3) reducing medical errors, and 4) improving patient safety and decreasing costs. Artificial intelligence has become commonly used in health-related decision support systems [7], numerous artificial intelligence techniques such as artificial neural networks [8].

In order to increase the diagnostic accuracy (DA) and decrease the cost, it is necessary to optimize the check combinations and achievement the check values fully. Till this point, with focusing on colorectal cancer (CRC), an artificial intelligent algorithm named DS-STM (diagnosis strategy of serum tumor makers) is still under development [9]. Association Rule Mining [10]. Genetic algorithms [11], the decision tree classification algorithm is a widespread cross-domain classification approach, used in problem domains such as marketing and customer preservation, fraud detection and medical diagnosis [12]. The ability of machine learning tools to detect significant features from complex datasets detects their importance. A variety of such techniques, including Artificial Neural Networks (ANNs), Bayesian Networks (BNs), Support Vector Machines (SVMs) and Decision Trees (DTs) have been applied in a wide manner in cancer studies for the development of predictive models, resulting in effective and accurate decision making [13], a low cost Smartphone based intelligent system incorporated with microscopic lens that lets patients in remote and isolated areas for uniform eye examinations and disease diagnosis. Mobile diagnosis system have been used an artificial Neural Network algorithm to analyze the retinal images taken by the microscopic lens to recognize retinal disease conditions [14].

\subsubsection{Some New types of IDSS}

With a view to adjust different situations, people improve the IDSS from various aspects and in different ways. This can be summarized as follows:

\subsubsection{Group Decision Support System (GDSS)}

It backings gathering or aggregate decision-making: it combines communication techniques, computer techniques and decision support systems, artificial intelligent techniques (AI) and thinking procedures, decision-making model methods, structuralization collective decision methods [15].

\subsubsection{Distributed Decision Support System (DDSS)}

This kind of backing is arranged not just toward individual decision-makers or any decision-making gathering that remain for the same organization, but also toward decision-making organizations that have separate character and are joined at the same time. It is impossible or in convenient to carry out some of the large-scale decision-making activity. These activities include decision-makers that have diverse responsibilities [16].

The data or important decision factors required for the decisionmaking procedure are distributed among a bigger area and this decision-making is a sort of association decision making or distributive decision-making.

\subsubsection{Intelligent, Interactive and Integrated Decision Support System (3IDSS)}

With the extension of the realistic field of DSS and the increasing of the application level, DSS has gone into the decision-making movement of regional medicinal and social advancement technique and the manufacturing and working decision-making of great-scale enterprises. Not just do these decision-making activities includes all the sides of medical activities and all the levels of management and administration, but also all the factors are associated with each other and the decision-making environment is problematical. Under this environment, a new comprehensive decision-making. DSS initiated existence, which is arranged toward decision-makers and the decision-making process. That is Intelligent, Interactive and Integrated DSS, abridged as 3IDSS [17].

\subsubsection{Intelligent Decision Support System based on Knowledge Discovery (IDSSKD)}

It is applied in commerce and finance market with the analysis, prediction and decision support. Generally speaking: IDSSKD = $\mathrm{DM}+\mathrm{WM}+\mathrm{KAS}+\mathrm{RM}$

In this formula, DM, namely data mining, mainly represents traditional structural data mining, in another word, KDD* system; WM (Web Mining) mainly includes text mining based on web, user access log mining and web structure mining; KAS (Knowledge Acquirement System with Intervention) is mainly used to acquire the knowledge of domain expert through induction; RM (Reasoning Machine) is mainly used when IDSS is carrying on reasoning. These four parts are combined through synthetic knowledge base and web knowledge base [18].

\subsection{Overview of Multi - Agent Systems}

Multi agent technology is applied by intelligent systems to solve the problems of analysis of complex systems and intelligent management activities. Multi agent system technology is a software paradigm where the concept of an agent denotes to "an encapsulated computer system that is located in some environment and that is capable of flexible, autonomous action in that environment in order to recognize its design objectives" [19].

Intelligent Multi Agent Systems (IMAS) based learning take in collection of information from their environment, recognition data, and intelligent classification data and forecast future data, storage data, delivery data to knowledge management systems such as Decision Support System (DSS) and Management Information System (MIS) [20].

A multi-agent system is functioned to automate processing within each step using agents with diverse abilities, and provides the parallelism of data processing using multi-agent system. The power of system is in its capability to support concurrent processing of gene data and the modular structural design that tailors to biologists' demands, also dispute the possibility of applying Machine Learning technique to anatomize gene expression data in a multi-agent environment [21]. Implement a homeopathic medical aid platform; first have to figure out how the general homeopathic medical diagnosis was done by a homeopathic practitioner. At the first stage the patient declared his/her infection (symptoms of illness) and also produced his former prescription or clinical or lab report if any. Next the initial diagnosis part where the signs of disease and several diagnoses were done. At the last stage (the final diagnosis part) based on the initial diagnosis and lab reports, the final diagnosis was done. After final diagnosis it was determined whether to generate 
formula for the patients or recognize the patient to a hospital, if his/her condition was critical [22]. The proposed hierarchical contains of service mobile agents as upper layer agent, coordinator agent as middle layer agent and initial agent as lowest layer agent. Coordinator agent helps as matchmaker agent that usages Naïve Bayesian learning method for gain general information and selects the best service supplier agent using matchmaking mechanism. Therefore this system can moderate the communication overhead between agents for sending messages and transferring data and can avoid sending the problem to unrelated agents [23].

\subsection{Review of Multi Agent Based on IDSS}

Recently, there is the absence of one united framework for integration of the two applicable flows of intelligent multi agent technology and IDSS in real environment. An overview over these agent-based methods and distributes them into five sections: Medical data management, decision support systems, scheduling and resource allocation, remote care as well as complex systems. Here, we focus on decision support systems that essentially aim at assisting professionals in their daily routine [24]. Agent is a procedure that can exchange the user to carry out a specific task individually and automatically. It can support the decision making and problem solving in various stages and also can enhance the functions of old-fashioned decision support systems. Multi-agent is loosely coupled network and has good dexterity and capability and it is used to form applications in a variety of areas and it can solve large-scale difficult problems through reciprocal scheduling and mutual cooperation among agents [25].

Application of evolutionary Multi-agent system (EMAS) to analysis of gene expression data to find considerable classification genes using simple classifier that can be used by agent when searching through the gene expression database search space, that agents improve their distinct performance through evolution and cooperation with other agents. Simple nearest neighbor classifiers are used in combination with evolutionary multi-agent based system [26].

Health Agents, European Commission (EC)-funded research project to improve the classification of brain tumors through multi-agent decision support in extra of a distributed network of local databases or Data Marts. Define a method to assess the quality and availability of a new candidate local database containing an arrangement of new cases, based on a compatibility score [27]. Ecological-medical situation assessment is using agent-based decision support system (ADSS). The system receives statistical information in form of direct and indirect pollution pointer values. The ultimate goal of the exhibited multiagent system (MAS) is to evaluate the power of the exposure to pollutants in population health [28]. A medical diagnosis system that combines the benefits of multi-agent system technologies and neural networks in order to realize a greatly reliable, adaptive, scalable, flexible, and robust diagnosis system for diseases. The medical diagnosis system consists of a structured alliance of medical experts - realized by agents - that cooperate in order to provide a sustainable medical diagnosis. Each agent has a certain responsibility. Reactive pattern-based matching process is the main support for the agents [29].

Modern research areas in medical that include intelligent decision support services, expert medical services and autonomous management are based on multi-agent systems. The assistance of these software agents offers efficient monitoring, analyzing, and managing the data of patient where abnormal patterns are detected to have an improvement treatment and avoid loss of life. The research presented a framework for ubiquitous healthcare based on multi-agent, proposes a mobile agent for diagnosis support in global healthcare. The expert mobile agent (EMA) categorize the data of patient by using neuro-fuzzy algorithm for consultation report. A pre-processing technique rely on the profile of an expert is used to as a filter of the patient history data. Results of neuro-fuzzy from cross-validation test shows a great accuracy in data classification compared to other highly accurate classifiers [30].

A multi-agent system developed in order to enhance gene expression analysis with the computerization of tasks about identification of genes involved in a cancer, and classification of tumors according to molecular biology. An agent that helps in system integrity, carry out reading files of data amount of genes from microarrays, information pre-processing, and with machine learning approaches make groups of genes involved in the process of a disease as well as the classification of samples that could propose novel subtypes of tumors hard to identify based on their morphology. Studies proved that the multi-agent system needs a minimal intervention of user, and the agents generate knowledge that decrease the time and complication of the work of preventing and diagnosis, and thus let a more effective treatment of tumors [31]. A multi agents system (MAS), which distributes the diagnosis on three agents. Each agent is a professional able to resolve and communicate with the other agents [32].

In vivo magnetic resonance spectroscopy (MRS) consolidated with in vitro multi-agent system (MAS) and gene expression promised to enhance the classification of brain tumours and produce novel biomarkers for prognosis. Health Agents was tended this issue by building a distributed system of databases centered on the clients and managed by agents. Thus, Health Agents proposes an exclusive blend of state-of-the-art technologies to advanced novel clinical tools for the diagnosis, management and understanding of brain tumours [33]. Decision algorithm is designed of decision tree and rough set. The method is applicable for cooperative decision among Agents and can help to whole complex works in Multi-Agent System. In the system, Agents can complete mutual decision practice of algorithm about cooperation and decision. As the Agent's own uncertainty, the effectual coordination requests to be solved between Agents for multi-Agent system [34].

An intelligent agent looks for circumstances that require informing healthcare professionals about events. These events can include giving shots, vaccinations, surgeries, follow-up checks and other essential events. Analyses of administrative data are the creator for these events as different to clinical data. The case study existing was tested on determining pneumonia vaccinations provider however it can be used in much greater areas [35]. Present an agent-based IDSS which is used for diagnosis includes three levels. The deepest level is called agents, which contains several kinds of agents including user interface agents, data mining agents and problem solver agents. The second level is communicators which act as translators among the task managers and the agents to ensure that every agent gets the information in their desired format. The upper level is task-managers, which break the task up into sub problems that are given to agents [36].

Studies utilized multi-agent IDSS about cancer gene study the system searches for information on several databases like PubMed keeping in mind the end goal to gather the most up and coming information as possible [37]. The EMG investigation framework is a data mining system that is utilized to mine electromyography (EMG) data the system contains three different agent types. These are the task agent which is the client interface agent, the sub-agent which has data around a specific part of anatomy and the data agent which is used to mine one around a specific field from one particular table [38]. The Clinical-HINTS 
system is DSS aimed to meet the requests for intelligent real-time clinical management in critical care medical environments for example, intensive care units (ICU). The system is a single agent based system where the agent is utilized to assess the data and stimulate any alarms that are required [39]. Intelligent agent proposed structure depends on neural network classification approach to solve the gap problem among two applicable flows of intelligent/ multi agent technology and the methodology of learning from real environment [20].

\section{COMPARISION BETWEEN MULTI- AGENT BASED IDSS}

Determining key practical necessities for later improvement of the multi-agent based IDSS, based on current multi-agents based IDSS research applied within the classification medical diseases was the purpose of this comparison framework, used to compare the research introduced in the previous section, was advanced using broad IDSS features, which include what category of decisions the systems support, making the given IDSS for the purpose of either decision speed or quality and decisions time sensitivity. The data subsystem was compared upon data medication features that at least one of the system tested supported. The data distributed or not there support for distributed data mining. The following features that were mentioned at minimum one of the examined systems can be compared to the agent subsystems. What are the languages of the agents coded in? The structure of the agents which is either a single agent or functional or layered multi-agent structures.

The user interface (UI) subsystem can be compared on numerous features, the utility of the user interface which is that it is either request/response where each request results is a reaction which can then drive to another request or an interactive UI where the person can justify and amend his requests at any time and the computer can make clarification request if required, what was the devices that UI available through.

This section presents the comparison of the study. The framework is first presented, and then the comparison of the research based on the framework. A discussion of the results of the comparison is then presented. The motivation of this comparison is to recognize open research questions, common system components, and the common structure of multi-agent based IDSS to further our research into multi-agent based IDSS for use cancer classification. The comparison of the study is shown in table 1.

Table 1. The Comparison between Frameworks

\begin{tabular}{|c|c|c|c|c|c|c|c|}
\hline $\begin{array}{l}\text { Paper } \\
\text { No. }\end{array}$ & $\begin{array}{l}\text { What does it } \\
\text { support? }\end{array}$ & $\begin{array}{l}\text { Is the aim to improve } \\
\text { decision speed or } \\
\text { decision quality? }\end{array}$ & $\begin{array}{l}\text { Is the data } \\
\text { distributed? }\end{array}$ & $\begin{array}{c}\text { What } \\
\text { Languages } \\
\text { were the agents } \\
\text { coded in? }\end{array}$ & $\begin{array}{l}\text { What is the } \\
\text { Agent } \\
\text { organizational } \\
\text { structure? }\end{array}$ & $\begin{array}{l}\text { What sort } \\
\text { of UI is it? }\end{array}$ & Diseases \\
\hline$[26]$ & Diagnosis & Decision Quality & No & Not Specified & Functional & $\begin{array}{c}\text { Not } \\
\text { Defined }\end{array}$ & $\begin{array}{l}\text { Colon } \\
\text { Cancer }\end{array}$ \\
\hline [27] & $\begin{array}{c}\text { Diagnosis/ } \\
\text { Prognosis }\end{array}$ & Decision Quality & Yes & Not Specified & Layered & $\begin{array}{c}\text { Not } \\
\text { Defined }\end{array}$ & $\begin{array}{l}\text { Brain } \\
\text { Tumors }\end{array}$ \\
\hline [28] & $\begin{array}{l}\text { Medical } \\
\text { Ecological } \\
\text { Assessment } \\
\end{array}$ & Decision Quality & Yes & Not Specified & Layered & Interactive & $\begin{array}{c}\text { Not } \\
\text { Defined }\end{array}$ \\
\hline [29] & Diagnosis & Decision Quality & Yes & Not Specified & Single & $\begin{array}{c}\text { Not } \\
\text { Defined }\end{array}$ & $\begin{array}{c}\text { Any } \\
\text { Diseases }\end{array}$ \\
\hline$[30]$ & Diagnosis & Decision Quality & Yes & Java & Mobile & $\begin{array}{c}\text { Not } \\
\text { Defined }\end{array}$ & $\begin{array}{c}\text { Heart } \\
\text { disease }\end{array}$ \\
\hline$[31]$ & Diagnosis & Decision Quality & Yes & Java & Functional & $\begin{array}{l}\text { Request/ } \\
\text { Response }\end{array}$ & Cancers \\
\hline [32] & Diagnosis & Decision Quality & No & Not Specified & Functional & Interactive & $\begin{array}{c}\text { Not } \\
\text { Defined }\end{array}$ \\
\hline$[33]$ & Diagnosis & Decision Quality & No & Not Specified & Layered & $\begin{array}{c}\text { Not } \\
\text { Defined }\end{array}$ & $\begin{array}{c}\text { Brain } \\
\text { Tumors }\end{array}$ \\
\hline [35] & Treatment & Decision Quality & Yes & Not Specified & Functional & $\begin{array}{l}\text { Request// } \\
\text { Response }\end{array}$ & $\begin{array}{c}\text { Not } \\
\text { Defined }\end{array}$ \\
\hline [36] & Diagnosis & Decision Quality & Yes & Not Specified & Layered & $\begin{array}{l}\text { Request/ } \\
\text { Response }\end{array}$ & $\begin{array}{c}\text { Not } \\
\text { Defined }\end{array}$ \\
\hline [37] & Research & Decision Quality & Yes & Not Specified & Single Agent & $\begin{array}{l}\text { Request/ } \\
\text { Response }\end{array}$ & $\begin{array}{c}\text { Not } \\
\text { Defined }\end{array}$ \\
\hline [38] & Diagnosis & Decision Quality & No & Java & Layered & $\begin{array}{l}\text { Request/ } \\
\text { Response }\end{array}$ & $\begin{array}{c}\text { Not } \\
\text { Defined }\end{array}$ \\
\hline [39] & Diagnosis & Decision Quality & No & $\mathrm{C}++$ & Single Agent & $\begin{array}{l}\text { Request/ } \\
\text { Response }\end{array}$ & $\begin{array}{c}\text { Not } \\
\text { Defined }\end{array}$ \\
\hline
\end{tabular}

\section{DISCUSSION}

The agent-based IDSSs studied cover the full scope of potential uses in treatment, diagnosis and research. The greater parts of the systems are deepened on enhancing decision quality instead of speed as they are either time insensitive. None of the agent-based IDSSs give feedback info about the data precision used to create decisions. Just few of the systems use distributed data but of those that do all of them are capable of distributed data mining.
Just four of the system descriptions contain what language was utilized to program the agents, which were $\mathrm{C}++$ and JAVA, this while not significant data is honestly significant for maintenance and upgrade purposes. For the most part, a considerable lot of the systems investigated had agent types specialized to work at different layers or for different purposes in the functional structured system situation. Two kinds of multi-agent IDSS from systems popular are layered as functional opposing. This is done 
to simplify the system and to make adjusting and improving the agents or the framework simpler

Most of the systems overviewed utilize a request/response system, which is where the system is given guidelines from the user and then gathers the necessary information and responds to the user and expects further commands. Just two uses an interacting system which is where the system might ask for or automatically provide clarification in a real-time style. None of the systems has any stand by forms of UI such as PDA, etc. Most of the systems examined dealt with closed MAS. These resources that the agents were intended to work cooperatively together simply and with few problems. So no conflict handling mechanisms were included, nor were any additional cooperation instruments for example auctions or negotiations included.

\section{CONCLUSION AND FUTURE WORK}

In this survey paper we have presented how multi-agent intelligent decision support system (MAS-IDSS) has been integrated in medical classification Problems to resolve various medical diagnosis and related problems. Still there are a few preferences, advantages and disadvantages of using MAS-IDSS in medical classification Problems. This survey paper aimed at providing the basic information and related work using multiagent, IDSS and MAS-IDSS system. No one of the designed techniques examined controlled time critical decisions or made try to reduce the decision time only to attempt to increase the decision accuracy. Most of the agent-based IDSSs examined checked support for distributed database except four systems not support distributed database mining. Only two of the systems collect data from any real-time data streams.

As future work, more agents will be designed and implemented for a multi-agent IDSS system to run in different machines, located in several diverse places and attempts to reduce the decision time and implementing more learning and statistical approaches to enhance and improve gene identification and cancer classification. Each of the agents may implicate of the learning required to solve the problem and add or delete these agents without affecting in the operation. Multi-agent IDSS systems offer a natural way of attacking distributed problems. Agents are able to communicate among themselves, using some type of agent communication language, in order that interchange any kind of information.

\section{REFERENCES}

[1] D. S. Kumar, G. Sathyadevi and S. Sivanesh," Decision Support System for Medical Diagnosis Using Data Mining”, IJCSI International Journal of Computer Science Issues, Vol. 8, issue 3, No. 1, Pages147-153, May 2011.

[2] A. B. AL-Badareen, M. H. Selamat, M. Samat, Y. Nazira and O. Akkanat," A Review on Clinical Decision Support Systems in Healthcare" Journal of Convergence Information Technology (JCIT), vol. 9, no. 2, pages125135, March 2014.

[3] B. L. Iantovics," Agent-Based Medical Diagnosis Systems", Computing and Informatics, vol. 27, no. 4, pages 593-625, 2008.

[4] D. Foster, C. McGregor and S. El-Masri," Survey of Agent-Based Intelligent Decision Support Systems to Support Clinical Management and Research", International Conference on Autonomous Agents and Multi-agent Systems, ACM, 2005.

[5] A. Kaklauskas, "Biometric and Intelligent Decision Making Support, chapter 2", Intelligent Systems
Reference Library, (c) Springer International Publishing Switzerland, Pages 31-85, 2015.

[6] S. Chakraborty, S. Gupta, "Medical Application Using Multi Agent System - A Literature Survey", Sougata Chakraborty et al Int. Journal of Engineering Research and Applications, Vol. 4, No. 1, pages 528-546, 2014.

[7] D. Bassen, S. Nayak, X. Chong Li and M. Sam," Clinical Decision Support System (CDSS) for the Classification of Atypical Cells in Pleural Effusions", Procedia Computer Science, Elsevier, Vol. 20, No. 2, Pages 379384, 2013.

[8] R.R.Janghel, A. Shukla, R. Tiwari and R. Kala, "Intelligent Decision Support System for Breast Cancer", Proceedings of the International Conference on Swarm Intelligence, Springer Lecture Notes in Computer Science, Beijing, China, Vol. 6146, No. 10, Pages351358, 2010.

[9] J. Shia, Q. Sud, C. Zhangb, G. Huangb and Y. Zhuc," An intelligent decision support algorithm for diagnosis of colorectal cancer through serum tumor markers", computer methods and programs in biomedicine, Elsevier, Vol. 100, Pages 97-107, 2010.

[10] C. W. Cheng, Ni. Chanani, J. Venugopalan, K. Maher, and M. D. WANG," icuARM An ICU Clinical Decision Support System Using Association Rule Mining", Translational Engineering in Health and Medicine, IEEE, Vol.1, No. 2, Pages 8-17, 2013.

[11] Aishwarya S and Anto S, "A Medical Decision Support System based on Genetic Algorithm and Least Square Support Vector Machine for Diabetes Disease Diagnosis", International Journal of Engineering Sciences \& Research Technology, IJESRT, Vol. 3, No. 4, Pages 4042-4046, April 2014.

[12] Y. Zhou, Y. Tan, H. Li and H. Gu, "A Multi-Classifier Combined Decision Tree Hierarchical Classification Method", International Symposium on Image and Data Fusion (ISIDF), IEEE, Pages1-3, 2011.

[13] K. Kourou, T. P. Exarchos, K. P. Exarchos, M. V. Karamouzis and D. I. Fotiadis, "Machine learning applications in cancer prognosis and prediction", Computational and Structural Biotechnology Journal, Elsevier, Vol. 13, No. 6, Pages 8-17, 2015.

[14] A. Bourouis, M. Feham, M.A. Hossain and L. Zhang," An intelligent mobile based decision support system for retinal disease diagnosis", Decision Support Systems, Elsevier, Vol. 59, No. 7, Pages 341-350, 2014.

[15] P. Gray, "The Nature of Group Decision Support Systems", Handbook on Decision Support Systems 1. International Handbooks Information System, pages 371389, 2008.

[16] A. Gachet," A New Vision for Distributed Decision Support Systems" Decision Making and Decision Support in the Internet Age, proceedings of the DSIage Conference, Oak Tree Press, Cork, Ireland, Pages 341352, 2002.

[17] Z. Mo, S. Feng, and C. Tang, "A Study on Integrated Model of Decision Support System", Journal of System s Science and System s Engineering, Vol. 11, No. 3, Pages 328-332, 2002. 
[18] F. Zhou, B. Yang, L. Li and Z. Chen," Overview of the New Types of Intelligent Decision Support System", the 3rd Intetnational Conference on Innovative Computing Information and Control (ICICIC'08), IEEE, Pages 267272, 2008.

[19] N. Jennings, "On agent-based software engineering", Artificial Intelligence, Vol. 117, No. 2, Pages277-296, 2000.

[20] R. Asadi, N. Mustapha, N. Sulaiman, "A Framework For Intelligent Multi Agent System Based Neural Network Classification Model", (IJCSIS) International Journal of Computer Science and Information Security, Vol. 5, No. 1, Pages 168-174, 2009.

[21] H.C. Lam, M. Vazquez Garcia, B. Juneja, S. Fahrenkrug and D. Boley," Gene Expression Analysis in Multi-Agent Environment",

http://citeseerx.ist.psu.edu/viewdoc/summary?doi=10.1.1 .109.2453.

[22] S. Chakraborty and S. Gupta,"A novel Multi Agent System based Homeopathic Medical Diagnosis System", CALCON 2014, IEEE, Pages1-4, 2014.

[23] E. E. Chaw," Naïve Bayesian Learning based Multi Agent Architecture for Telemedicine", International Journal of Innovation and Applied Studies, Vol. 2, No. 4, Pages 412-422, Apr. 2013.

[24] D. Isern, D. Sánchez, and A. Moreno, "Agents applied in health care: A review", International Journal of Medical Informatics, Elsevier, Ireland, Vol. 79, No. 3, Pages145166,2010 .

[25] S. Yongyong," decision algorithms for Multi-agent Intelligent Decision Support System based on blackboard", Information Technology Journal, Vol. 12, No. 21, Pages 6235-6240, 2013.

[26] G. Stiglic, P. Kokol, "Colon cancer prediction with genetics profiles using evolutionary techniques", Expert Systems with Applications: An International Journal, Vol. 38, Issue 3, ACM, Pages 2752-2757, 2011.

[27] H. Gonzalez-Velez, M. Mier, C. Arus, B. Celda, S. V. Huffel, P. Lewis, A. Peet and M. Robles," Agent-Based Distributed Decision Support System for Brain Tumour Diagnosis and Prognosis", Applied Intelligence, Spring Link, Vol. 30, No. 3, Pages191-202, June 2009.

[28] M. V. Sokolova and A. F. Caballero, "An Agent-Based Decision Support System for Ecological-Medical Situation Analysis", Nature Inspired Problem-Solving Methods in Knowledge Engineering Lecture Notes in Computer Science, Vol. 4528, Pages 511-520, 2007.

[29] C. Klüver, J. Klüver and R. Unland," A Medical Diagnosis System based on MAS Technology and Neural Networks", BPSC, vol. 147, Pages179-191, 2009.

[30] R. M. A. Mateo, L. F. Cervantes, H. Yang and J. Lee,“ Mobile Agents using Data mining for Diagnosis Support in Ubiquitous Healthcare", Agent and Multi-Agent Systems: Technologies and Applications Lecture Notes in Computer Science, Springer link, Vol. 4496, Pages795-804, 2007.

[31] E. Marquez, J. Savage, C. Lemaitre, A. L. LaureanoCruces3, J. Berumen, A. Espinosa, R. Leder and A. Weitzenfeld," A Decision Support System Based on Multi-Agent Technology for Gene Expression Analysis", International Journal of Intelligence Science, Vol. 5, Pages158-172, 2015.

[32] O. Kazar, Z. Sahnoun and L. Frecon," Multi-agents system for medical diagnosis", International Conference on Intelligent System and Knowledge Engineering, Vol. 1, Pages1265 - 1270, 2008.

[33] C. Arus, B. Celda, S. Dasmahaptra and D. Dupplaw," On the Design of a Web-Based Decision Support System for Brain Tumour Diagnosis Using Distributed Agents", International Conference, Web Intelligence and Intelligent Agent Technology Workshops, IEEE/WIC/ACM, Pages 208 - 211, 2006.

[34] S, Yongyong," The Realization on Cooperative Decision in Multi-Agent Intelligent Decision Support System", International Journal of Advancements in Computing Technology(IJACT), Volume 5, No. 6, Pages 998-1007, March 2013.

[35] C. Coffin, C. Saunders, C. Thomas, A. Loewen, N. Campbell and William Ghali," Using Intelligent Agents to Repurpose Administrative Data in Fostering Disease Prevention in an Outpatient Context: The Case of Pneumococcal Vaccination", Proceedings of the 37th Hawaii International Conference on System Sciences, IEEE, Pages1-7, 2004

[36] M. E. Cohen, D. L. Hudson," Meta Neural Networks as Intelligent Agents for Diagnosis", Neural Networks, IJCNN '02. Proceedings of the International Joint, IEEE, Vol. 1, No. 3, Pages 233 - 238, 2002.

[37] S. Z. H. Zaid, S. S. R. Abidi and S. Manickam,' Leveraging Intelligent Agents for Knowledge Discovery from Heterogeneous Healthcare Data Repositories", US National Library of Medicine National Institutes of Health, , Vol. 90, Pages 335-340, 2002.

[38] J. Balter, A. Labarre-Vila, D. Ziebelin, C. Garbay," A knowledge-driven agent-centred framework for data mining in EMG", US National Library of Medicine National Institutes of Health, C R Biol, Vol. 325, No. 4, Pages375-82, 2002.

[39] D. Kalogeropoulos, E.R. Carson and P.D. Collinson," Clinical-HINTS: Integrated Intelligent ICU Patient Monitoring and Information Management System", US National Library of Medicine National Institutes of Health, Stud Health Technol Inform, Vol. 43, 1997, Pages906-1010. 